\title{
The pros and cons pertaining to tuberculosis and tuberculosis protocols
}

\section{Rishan Singh}

University of KwaZulu-Natal. Durban, 4001. South Africa. Email: rshnsingh1@webmail.co.za.

\begin{abstract}
There are many pros and cons with the protocols used to diagnose patient with TB, particularly because research isn't able to correlate findings with the virulence of the tuberculosis pathogen. This has resulted in further experiments to try optimise protocols for better evaluation of susceptible and resistant tuberculosis strains. This indicates the need for more health-care facilities to treat patients with tuberculosis, or cotuberculosis infections, more precisely. This article presents some advantages and disadvantages of tuberculosis treatment protocols, with emphasis on drugs, tuberculosis data sets and the administration of plant compounds. This paper also highlights some points on the mechanism of drug action with tuberculosis. Controversies and the present status of tuberculosis studies are also commented on. This is the first (generalised) paper to highlight laboratory as well as nonlaboratory points pertaining to tuberculosis and tuberculosis protocols.
\end{abstract}

Keywords: Mycobacterium tuberculosis; Antimicrobials; Inhibitory; Stimulatory; Immunosuppression; Virulent; Immunocompromised; Pathogenesis; Cytology; Susceptibility; Resistance.

\section{Introdução}

Tuberculosis (TB) is a lung disease caused by the bacterium Mycobacterium tuberculosis (Singh, 2013). This disease is characterised by the coughing of blood as well as the difficulty in breathing in infected patients (Singh, 2011; Singh, 2013). Tuberculosis is often acquired by the inhalation of respiratory droplets when a no-diseased person comes I contact with it from coughing patients. However, the droplets may be transmitted in the air distant to an infected or unaffected person (Singh,
2018). Now, it has become easy to diagnose this disease, specifically because laboratory tests such as inoculation and isolation of the bacterium can be achieved (Singh, 2013). Tuberculosis, however, is a deadly disease if the patient isn't treated on time, and the severity of symptoms i.e. patients are usually seen as progressive, particularly because of the invasive nature of this disease (Singh, 2018). Furthermore, because the bacterium multiplies during aerobic respiration, the virulence of the bacterium, if untreated, overtime, manifests with severe 
symptoms (Singh, 2011). Although three kinds of antimicrobials are present in and in the treatment of tuberculosis, if the disease has progressed, treatment can be restricted to the use of a combination of these drugs (Singh, 2013). Since these drugs would not be discussed in this article, it's imperative to understand that each drug used in the treatment of tuberculosis is specifically designed to target a particular cell signalling system, which is often governed by protein regulatory enzymes (Singh, 2018). However, severe flu-like symptoms are often the initial prognosis by medical practitioners, other drugs may also be used to try treat the disease, however, this is only in the case of misdiagnosed patients (Singh, 2011; Singh, 2013; Singh, 2018).

\section{Problems associated with tuberculosis treatment protocols}

\section{Drugs and strains}

The problems with TB tests are varied, and differ between laboratory and regions from where the sputum is collected from patients (Singh, 2013). One of the problems is that it's difficult to produce results that are $100 \%$ conclusive in the laboratory (Singh, 2013). Although published reports slow consistent results, which are indeed more or less representative of a laboratory task, the diagnosis or presentation of first, second or third line drugs i.e. patient-identified cases are indeed problematic (Humphries and Hindler, 2016). It has been demonstrated in the literature that although sputum may be of multidrug resistant bacterium, during experimentation, additional resistance may occur (Alexander and Strete, 2001; Humphries and Hindler, 2016). This is a problem associated with TB treatment protocols in that it indicates an unavoidable cost of having to re-screen identified tuberculosis strains, indicating that possibly new drugs need to be manufactured and introduced to consumers (Singh, 2018). This means that when multidrug resistant bacteria become extremely drug resistant to a particular drug compound, perhaps the new drug compound contributes to an additional resistance (Singh, 2011; Singh, 2018). This Is an unavoidable disadvantage, particularly, since bacterium continuously multiply in susceptible hosts until an administered drug, or combination of such, has an effect, if any, on the infected bacterium strain (Victor et al., 1997; Petrini and Hoffner, 1999; Alexander and Strete, 2001; Humphries and Hindler, 2016; Singh, 2018).

\section{Presentation of data sets}

Data sets aren't really a problem in tuberculosis research (Singh, 2013). However, they can be misinterpreted if, and when, they are nor presented properly. Preliminary findings are important in scientific papers about tuberculosis, as it's able to indicate distinctly to researchers about the drug therapy that could possibly work for infected strains of the bacterium (Singh, 2011; Singh, 2018). This means that although prognosis and diagnosis have now become well known by practitioners, data sets could still be problematic (Singh, 2011). The correct way of forming a prognosis of a patient would be to compare resistant tuberculosis strains with control tuberculosis strains that have been exposed to the same treatment (Singh, 2018). In this way susceptibility patterns can be achieved for the tuberculosis strain that represents particular patients. The true nature of such results is that single drugs can be used as a starting point for treatment, followed by drugs that are wither more or less toxic than the one already administered (Victor et al., 1997; Jenkins and Jerris, 2011). Additionally, a turbidity standard needs to be used at all times, so that the bacterium cultures spread out on agar plates, for example, are of similar concentration constituency (Singh, 2018). However a problem, here, is that 
the viability of the bacterium culture would indeed be inconsistent, in that the multiplication rate of individual strains may differ (Singh, 2018). Another problem is that due to this set back, individual drugs may vary within individual culture plates for single strains. This is not always the obvious situation in the case of reported findings, particularly because this point is often substantiated or remains as preliminary findings (Singh, 2011; Singh, 2013). Another problem reported in the scientific literature is that often data sets are presented randomly (Singh, 2018). This means that comparisons are lost, and therefore cannot be commented on adequately. However, this does not mean that though they may be performed with the best precision as possible (James and Jorgensen, 2009; Singh, 2013). Therefore, it is recommended that data sets be presented in rows and separated in columns by the control strain, $\mathrm{H}_{37} \mathrm{R}_{\mathrm{v}}$, so that unavoidable errors can be avoided at a minimum (Singh, 2018).

\section{Administration of plant compounds}

The administration of plant compounds isn't as problematic if preparations are accurate (Singh, 2018).However, the problem associated with tuberculosis treatment protocols with plant compounds are many because of the conglomerate of substances contained within preparations (Singh, 2013). The problem in the protocols are of 4 types: 1) the interaction of the plant compound with the bacterial wall (Singh, 2013); 2) the probable interruption of compound entry due to external structures, such as pili (Victor et al., 1997; Petrini and Hoffner, 1999); 3) The mucus layer over the capsule and the capsule itself making it difficult for administered plant compounds to enter (Petrini and Hoffner, 1999), and 4) the interaction of the solvent used with the external and internal structures of the bacterium (Singh, 2013). Here, it is evident that a lot of work still needs to be done to correctly understand what happens when a plant compound enters a bacterium. Furthermore, as this is the case, it's evident that the same plant compound, whether isolated or in the form of an extract, would have a differed response in different bacterium strains. This means that the events leading up to a bacterium cell arrest would be different, indicating that it would be difficult for a medical practitioner to make an accurate diagnosis for any one isolated/administered plant compound (Singh, 2013). Also, like in cancer studies, individual plant compounds would either stimulate or arrest mycobacterium growth since plant compounds contain cell inhibitory and stimulatory compounds (Singh, 2018; Singh, 2013). However, in some instances, a problem may arise due to the viability of mycobacterium, where it may be found that a plant stimulatory compound may actually inhibit its growth; and vice versa (Singh, 2013).

\section{A controversy with tuberculosis bacilli}

A major controversy with tuberculosis bacterium is that many think that tuberculosis being air-borne, can be transmitted long distance from infected hosts. As mentioned, although this is a possibility, the likelihood of this happening is slim (Singh, 2013). This means that although tuberculosis is an endemic disease (Wolinsky, 1993) with very high incidences in South Africa and Hong Kong being recorded, its far spread isn't possible (Singh, 2013). Perhaps this could be due to the evaporation of infected respiratory droplets through the process of transpiration (Singh, 2013). Although this isn't a reported fact in medical terms, it's possible since these droplets are far too small to be interspersed through the atmosphere. However, since tuberculosis is tube-like and bacilli in shape, even the inhalation of a small droplet can trigger an automated immune response (Singh, 
2013; Singh, 2018). However another controversy is that tuberculosis bacilli may be destroyed in immune-strong hosts, while the converse is that since these bacteria are virulent, they may also spread far enough and kill the host (Singh, 2018). Although the latter statement isn't controversial, it's not completely certain whether respiratory processes in mammalian hosts support the multiplication of tuberculosis bacteria, however it's known that tuberculosis can only be viable in susceptible/living hosts with or without immunosuppression caused by a bacterial strain, virus or drug (Singh, 2011; Singh, 2013; Singh, 2018).

\section{The internal mechanism of drug action with tuberculosis}

The internal mechanism of drug action with tuberculosis is very much different in comparison to tissue culture cells. This is because the structure of TB bacterium are very different to tuberculosis bacteria (Singh, 2013). The mechanism of drug action differs slightly between susceptible, resistant and extremely drug resistance strains, but ultimately upon killing tuberculosis microbes, the process is no different. There are 5 steps of drug action in attempting to arrest tuberculosis (in patients with other virulent diseases like HIV for example). First the compound must interact with external structures of the bacterium, secondly it must transverse into the bacterium cell wall and capsule, thirdly it must enter the cytosol and interact with structures responsible for phagocytosis, fourthly the compound must disrupt the nucleois and initiate a signal of cell arrest, and lastly the waste products must be engulfed by macrophages (Singh, 2011; Singh, 2013; Singh, 2018). Once the last step occurs in infected patients, it can be deduced that the internal mechanism of drug action has been successful. Though this isn't the only 5 factors for successful drug action in a mycobacterium, it is the 5 paramount steps of successfully ridding infected patients with susceptibility, or less drug-resistant, strains of mycobacterium (Singh, 2013). In the event of the last factor not being successful once a drug had been administered to a tubercle bacilli, then this is a probable indication of inaccurate laboratory protocols (read Jenkins and Jerris, 2011). Though the protocols used to initiate an internal cascade of cell death in tuberculosis bacterium, in infected hosts, may be optimum, the internal host and bacterium conditions ultimately have a profound influence on the state of tuberculosis bacterium tested with compounds (Singh, 2013).

\section{Comment on the present status of tuberculosis studies}

The present status of tuberculosis studies is that a lot of new information about the protocols used to test susceptibilities have been found. However, in South Africa and overseas, a limiting factor has been the inability to adequately understand the virulence and pathogenesis of this bacterium outside medical terms (Singh, 2013). However it has become an interesting laboratory endeavour to relate mycobacteria susceptibility of drug compounds to resistance in tuberculosis studies in SA and other countries (Victor et al., 1997; Jenkins and Jerris, 2011; Humphries and Hindler, 2016). More information about the techniques used to assay drug compounds on tuberculosis wouldn't necessarily improve diagnostic protocols, without proper optimisation protocols being put in place (Wolinsky, 1993; Singh, 2013). Another downfall is that the interaction of drug compounds with mycobacteria isn't ravelling new information about the enzymatic nature of cell death. However, the advantage, here, is that new information about the cytotoxicity of these mycobacteria are being placed on record. This is an indication that the present status of 
tuberculosis is promising (Singh, 2013; Singh, 2018).

\section{Conclusions and perspective}

Tuberculosis is a disease that is transmitted in the air. However, it's possible that in non-well-ventilated facilities, not necessarily in rural areas only, nosocomial transmissions are also prevalent. Although tuberculosis is an endemic disease, its transmission isn't restricted to a particular age group or section of society. However immunosuppressed as well as immunocompromised hosts are those most susceptible of acquiring this disease. The laboratory protocols used to test the susceptibility and/or resistance of Mycobacterium tuberculosis isolates, or innoculums, are also well established inspite of a clear understanding of the bacterium nor being fully grasped by researchers. This means that in the fight for proper health care facilities to treat tuberculosis patients, an understanding of the bacterium together with external bacterium features and the environment is pivotal to make an accurate patient diagnosis and prognosis. Though this paper focussed on tuberculosis, its controversies and protocols, the points states in this article are of a broader scope, and thus, forms the basis of, and for, all prokaryote and eukaryote cells, particular since the environment is inclusive of both cell types.

\section{Conflicts of interest}

Author declares that they have no conflict of interests.

\section{References}

Alexander, S. K.; Strete, D. Microbiology: A photographic atlas for the laboratory. USA: Benjamin Cummings, Addison Wesley Longman Inc., 2001.
Humphries, R. M.; Hindler, J. A. Emerging resistance: new antimicrobial agents - but no test! The challenge of antimicrobial susceptibility in the current US respiratory landscape. Clinical Infectious Diseases, v. 63, no. 1, p. 83-86, 2016. https://doi.org/ $10.1093 / \mathrm{cid} / \mathrm{ciw} 201$

James, H.; Jorgensen, J. Antimicrobial susceptibility testing: A review of general principles. Clinical Infectious Disease, v. 49, no. 11, p. 1749-1755, 2009. https://doi.org/ $10.1086 / 647952$

Jenkins, S.; Jerris, R. Critical assessment of issues applicable to development of antimicrobial susceptibility testing breakpoints. Journal of General Microbiology, v. 10, p. 49-55, 2011. https://doi.org/10.1128/JCM.00837-11

Petrini, B.; Hoffner, S. Drug-resistant and multidrug resistant tubercle bacilli. International Journal of Antimicrobial Agents, v. 13, p. 93-97, 1999. https://doi.org/10.1016/S0924-8579(99) 00111-9

Singh, R. Minimum inhibitory concentrations: Interpretation and cross-sectional analysis in an unstandardized 7H9 Mycobacterium tuberculosis broth-based system: A hypothetical case. Journal of Pure and Applied Microbiology, v. 7, no. 1, p. 777784, 2013.

Singh, R. Mycobacterium tuberculosis: Telling a story of truths. Journal of Pure and Applied Microbiology, v. 5, no. 1, p. 541544, 2011.

Singh, R. personal writing. Representing the Republic of South Africa, my country, 2018.

Starr, R.; Taggart, R. Biology: The unity and diversity of life. 9. ed. Brooks/Cole Publication, 2001.

Victor, T. C.; Warren, R.; Butt, J. L.; Jordaan, A. M.; Felix, J. V.; Venter, A.; Sirgel, F. A.; Schaaf H. S; Donald, P. R.; Richardson, M.; Cynamon, M. H.; van Helder, P. D. Genome and MIC stability in Mycobacterium tuberculosis and indications for continuation of use of isoniazid in multidrug-resistant tuberculosis. Journal of Medical Microbiology, v. 46, p. 847-857, 1997. https://doi.org/10.1099/ 00222615-46-10-847 
Wolinsky E. Statement of the tuberculosis committee. Infectious Disease Society of America, v. 16, p. 627-626, 1993. 